\title{
Experimental investigation of magnetic islands growth in tokamak plasmas by applying emissive biased limiter and resonant helical field
}

\author{
A Aslani and M N Nasrabadi \\ Faculty of Physics, University of Isfahan, Isfahan, Iran \\ E-mail: mnnasrabadi@ast.ui.ac.ir
}

(Received 16 January 2019 ; in final form 22 July 2020)

\begin{abstract}
At the present time, Magnetic Confinement Fusion $(M C F)$ is considered as a way to produce energy. In this work, one of the Magnetohydrodynamic (MHD) limitations has been discussed. As closed magnetic surfaces, the Magnetic Islands (MIs) which are generated due to pressure effects, need to be surrounded by a separatrix which separate them from the other parts. External Magnetic Fields $(E M F s)$, the safety factor $(q)$ and the pressure profiles would be used to take the MIs under control. This could be achieved through an exterior medium, exclusively the Electron Cyclotron Heating $(E C H)$ as well as the Current Drive $(E C C D)$. Study of the magnetic flux surfaces and the effect of magnetic perturbation on tokamak plasmas, inform us about the formation of the MIs and their locations. In this work, together with the comprehensive review of the MIs and their importance, the conventional methods for improving the magnetic confinement has been introduced and discussed. In this regards, the Hot Limiter Biasing $(\mathrm{H} L B)$ method and the Resonant Helical Field $(R H F)$ which is produced by external Helical Coils $(H C s)$ were introduced and used. Then, the plasma current $\left(I_{p}\right)$, the Loop voltage, and the $M O$ were obtained for different states. Finally, the Magnetic Islands Width (W) and their Growth Rate $(G R)$ were calculated and compared with the experimental results.
\end{abstract}

Keywords: tokamak, magnetic islands, separatrix, limiter biasing, resonant helical field

\section{Introduction}

MHD instabilities play an important role in the plasma disturbances. These instabilities lead to the destruction of the magnetic flux surfaces and the appearance of plasma discharge. In tokamaks, taking these phenomena under control is of crucial importance. Some experimental results have indicated that correction and confinement improvement can be performed in a controlled manner by generating an induced Electric Field $(E F)$ at the plasma edge using $L B$ or through the $R H F$ that enters the main field as a perturbation. The resistive tearing modes have been claimed to be another type of $M H D$ instabilities which are responsible for the disturbances in the plasma. These modes lead to the breakdown of the magnetic flux surfaces and organize the helical structures which are referred to as MIs which are radially localized magnetic structures [1]. These modes imply serious minor and major disruptions on the plasma confinement. The major disruptions are capable to cease the plasma discharge [2]. In practice, the tearing modes affect resonant magnetic surfaces $(R M S)$ having rational quantities of the safety factor $q$. There are many data processing techniques and diagnostics which are able to analyze the oscillations due to the tearing modes in tokamaks, like Mirnov Oscillations (MO) [3] and Sawtooth oscillations [4]. These oscillations can be illustrated by temporal evolutions and spatial structures determined by the poloidal and toroidal mode numbers which are given by $m$ and $n(m, n)$ respectively. In most of tokamaks, it is seen that the interaction of $(2,1)$ mode with the limiter or the cold plasma at the edge leads to the major disruptions [5]. Since the major disruptions could be attributed to the $(2,1)$ mode, then the development of $(2,1)$ mode will be useful if a perturbed $M F$ generated by the Resonant Helical Coil (RHC) is applied [6].

The RHF affected by the RHCs may strongly affect the MIs on the $q=2$ resonance surface in Tokamaks. It should be noted that $W$ might be efficiently diminished by the RHF by the $M O$ [7]. Consistent with the recent experiments, the $M O$ might be dramatically inhibited due to mode stabilization in low- $q$ and low-density plasmas 
[8]. In practice, it is possible for the mode stabilization to occur, once the rotating frequency of the MIs is diminished while applying the $R H F$. It should be noted that there is a correlation between the decreases in the mode frequency and decreases in mode amplitude instead of the $R H F$ amplitude. The $L B$ experiment with positive polarities in HT-7 tokamak shows that by applying the $L B$, the particle confinement will be improved with a factor about 1.5 and $M H D$ oscillations are reduced in the flat part of the plasma flow during disruption and at the time of the decreases in the plasma flow. There is a relationship between the induced $E F$ constructed by $L B$ and modification in $M H D$ activities in several experiments. Since the $L B$ flow is low in negative polarities, it has no effect on the improvement of the plasma parameters. The spatial and temporal variations of the modes in IR-T1 tokamak are based on $M O$. Using the Fast Fourier Transform (FFT) analysis technique, the $L B$ and the $R H F$ are investigated. In this work to improve the plasma oscillations, two methods were used. This work somehow developed the previous investigations on the effect of $H L B$ and $R H F$ on the plasma parameters and fluctuation of the $M F$ around the IR-T1 chamber. To do this, an array of 12 external Mirnov Coils (MCs) located poloidally by $30^{\circ}$ was employed (Fig. 1).

The plasma mode numbers were determined utilizing the FFT [9-11]. Moreover, in order to measure the plasma parameters, some diagnostics such as magnetic probe and a poloidal flux loop as well as the Rogowski Coil $(R C)$ were employed. To enhance the plasma oscillations, two methods were used. In the first one, the plasma modes and parameters were investigated in the lack of $H L B$ and $R H F$ and in the second one they were investigated in the presence of $H L B$. In addition, an experimental estimation of $R H F$ effects on the plasma modes and parameters was reported.

\section{Experiment}

The experimental results show that the improved confinement is related to the development of strongly radially varying profiles of the $E F$. The results show that $E_{r}$ profile, reduces the anomalous transport. A theoretical method is also presented to justify the relationship between reduced transport and the sheared velocity, $E \times B$ due to Radial Electric Field $\left(E_{r}\right)$ oscillations. The $E F$ shear can also modify the classical and neoclassical transports by a process known as orbit squeezing which can be shown for Larmor orbit in a uniform $M F$ in the $Z$-direction. Consider the Hamiltonian of a particle moving in a uniform $M F$ and a quadratic electrostatic potential well. Then,

$$
\begin{aligned}
& \Phi(x)=\frac{x^{2}}{2} \Phi^{\prime \prime} \quad\left(\Phi^{\prime \prime}>0\right), \\
& H=\frac{m}{2} P_{\mathrm{x}}^{2}+\frac{m}{2}\left(P_{y}-e B \mathrm{x}\right)^{2}+\frac{m}{2} P_{z}^{2}+\frac{e \Phi^{\prime \prime} \mathrm{x}^{2}}{2},
\end{aligned}
$$

In which $p_{x}, p_{y}, p_{z}$ are the canonical momentums with $p_{y}$ and $p_{z}$ as conserved quantities. Then, the motion of a particle in the potential well and in the $x$-direction, leading to an excursion about $x=0$, giving the usual Larmor radius. $E_{r}$ satisfies the radial force balance equation for any ion of charge $\mathrm{Ze}$.

$$
E_{r}=\frac{1}{n Z e} \frac{d p_{i}}{d r}-V_{\theta i} B_{\phi}+V_{\phi i} B_{\theta} \text {, }
$$

where $V_{\theta i}$ and $V_{\phi i}$ refer to the poloidal and toroidal components of ion velocity respectively. On the other side, $E \times B$ (related to drift velocity) can be generated by applying a voltage using the biased probes at the plasma edge. Equation (3) shows that they can also be produced indirectly in another ways: applying additional heating to increase the ion pressure gradient using momentum inputs to drive $V_{\theta i}$ and $V_{\phi i}$ against viscous

damping; and by the flows driven by the turbulence itself through the Rynolds stress. Computational simulations of the plasma turbulence indicate that the fine scale poloidal $E \times B$ current can be generated by the turbulence. These are called zonal flows and are found to reduce the saturated level of the turbulence. To achieve controlled thermonuclear fusion, it is required that tokamaks to be operated in a High-Confinement mode (H-mode), and this was considered in ASDEX tokamak using additional heating (NBI) in 1982. Practically, little is known about the fundamental physics of the transition from the Low- Confinement mode (L-mode) to the $\mathrm{H}$ mode, although most of tokamaks are operated in $\mathrm{H}$ mode. Based upon numerous experiments, $E_{r}$ is crucial in this transition. This issue was verified through an experiment was carried out in the CCT tokamak. In this experiment, to reach the $\mathrm{L}-\mathrm{H}$ transition, an externally biased electrode in the outer edge region of the tokamak was introduced. Thereafter, the electrode biasing has turned into the commonest means for investigating $\mathrm{L}-\mathrm{H}$ transitions in smaller tokamaks with the limiter/divertor configuration. The sheared $E_{r}$ model increases the local shear flows which in turn lead to cessation of the electrostatic turbulence in order to reach the $\mathrm{H}$-mode regimes. Generally, $L B$ has been acted successfully in confinement by setting up a $E_{r}$ at the plasma edge. In this method by applying $E_{r}$ which interacts with the $M F$, the natural modes of the plasma can be enhanced. The $H L B$ is shown schematically in fig. 2 .

In order to enhance $L B$ a tungsten filament was used as a heater. $H L B$ is able to emit electrons into the plasma. The experiments were performed using hydrogen under given conditions in table 1 , in which $T F$ and $D D$ refer to Toroidal Magnetic Field and Discharge Duration respectively.

Various plasma fundamental parameters including $M O$ and the loop voltage, as well as $I_{p}$ were investigated. It was confirmed that the $M C s$ are able to describe the current time series excited by the poloidal rotation of plasma. The polar diagram related to the $M F$ oscillations was compiled using $F F T$ analysis on a poloidal array of the $M C s$ (Fig. 3). The results show the plasma crosssection at three intervals while, $H L B$ was neglected. The $1^{\text {st }}$ time interval was $20-21 \mathrm{~ms}$, demonstrating $m=4$. 


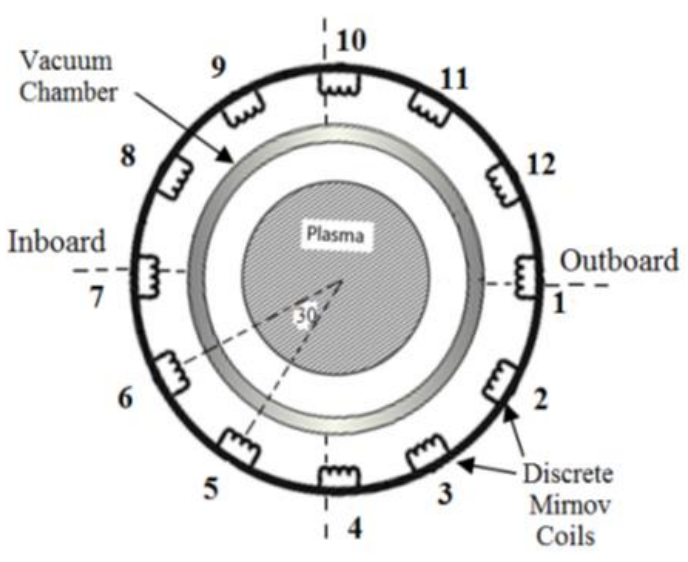

Fig. 1. Poloidally array of $12 \mathrm{MCs}$.

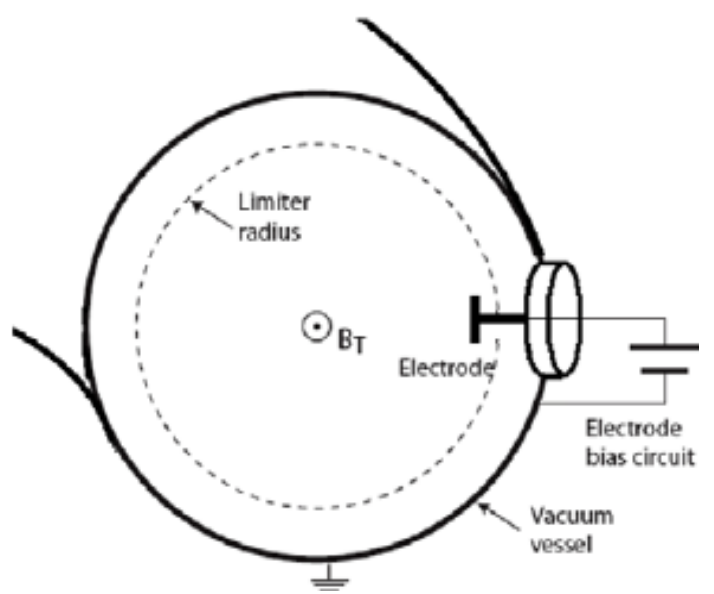

Fig. 2. Schematic of a HLB system.

Table 1. Plasma and field parameters in the IR-T1 Tokamak.

\begin{tabular}{c|ccccccc}
\hline Parameters & $\mathrm{R}(\mathrm{m})$ & $\mathrm{a}(\mathrm{m})$ & $\mathrm{TF}(\mathrm{T})$ & $\mathrm{IP}(\mathrm{kA})$ & $\mathrm{n}_{\mathrm{e}}\left(\mathrm{cm}^{-3}\right)$ & $\mathrm{DD}(\mathrm{ms})$ & $\tau_{\mathrm{c}}(\mathrm{ms})$ \\
\hline Values & 0.45 & 0.15 & $0.6-0.8$ & $25-30$ & $1-2 \times 10^{13}$ & 30 & $0.5-3$ \\
\hline
\end{tabular}
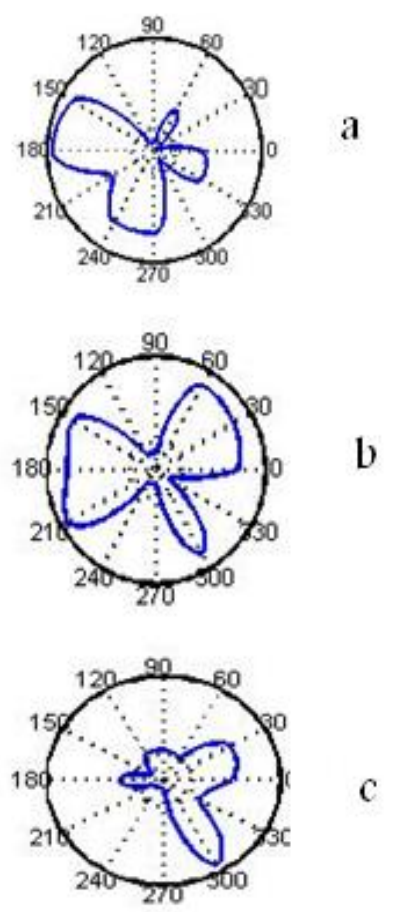

Fig. 3. Cross sections of plasma without $E B L$ and $R H F$ : a) in the range $20.0-21.0 \mathrm{~ms}$ with $\mathrm{m}=4$; $\mathrm{b}$ ) in the range $24.0-25.0$ $\mathrm{ms}$ with $\mathrm{m}=3$; $\mathrm{c}$ ) in the range $27.0-28.0 \mathrm{~ms}$.

Moreover, the $2^{\text {nd }}$ interval was $24-25 \mathrm{~ms}$ showing $m=3$ whereas the $3^{\text {rd }}$ interval was $27-28 \mathrm{~ms}$ showing $m=4$. The present work expresses the plasma cross section by considering that $H L B$ having a positive voltage, as shown in fig. 4. It is known that the plasma would remain in steady state if the quantity of $m$ remains less than 3 . The presented plots reveal that the mode numbers of plasma could be enhanced by introducing $H L B$ in the plasma edge of Tokamak. The plots introduce the time
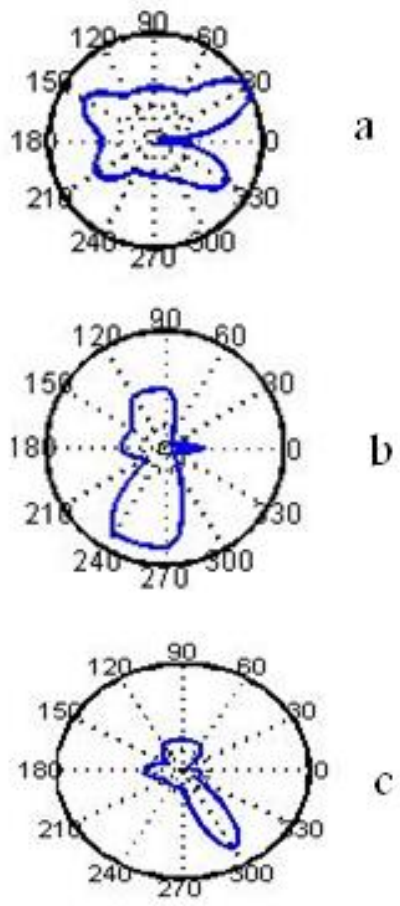

Fig. 4. Cross sections of plasma with $E B L$ : a) in the range $20.0-21.0 \mathrm{~ms}$ with $\mathrm{m}=4$; b) in the range $23.0-24.0$ $\mathrm{ms}$ with $\mathrm{m}=4 ; \mathrm{c}$ ) in the range $27.0-28.0 \mathrm{~ms}$.

evolution of main plasma parameters with and without $H L B$.

Fig. 5 shows $I_{p}$, loop voltage, and $M O$ in the absence of $R H F$ and $H L B$. Furthermore, fig. 6 shows $I_{p}$, loop voltage, as well as the $M O$ in the presence of $H L B$. It can be seen, $I_{p}$ is more uniform in $H L B$ state than normal and it is identified that $I_{p}$ remains flat after applying the $H L B$ at $\mathrm{t}=10 \mathrm{~ms}$. In addition, the amplitude 

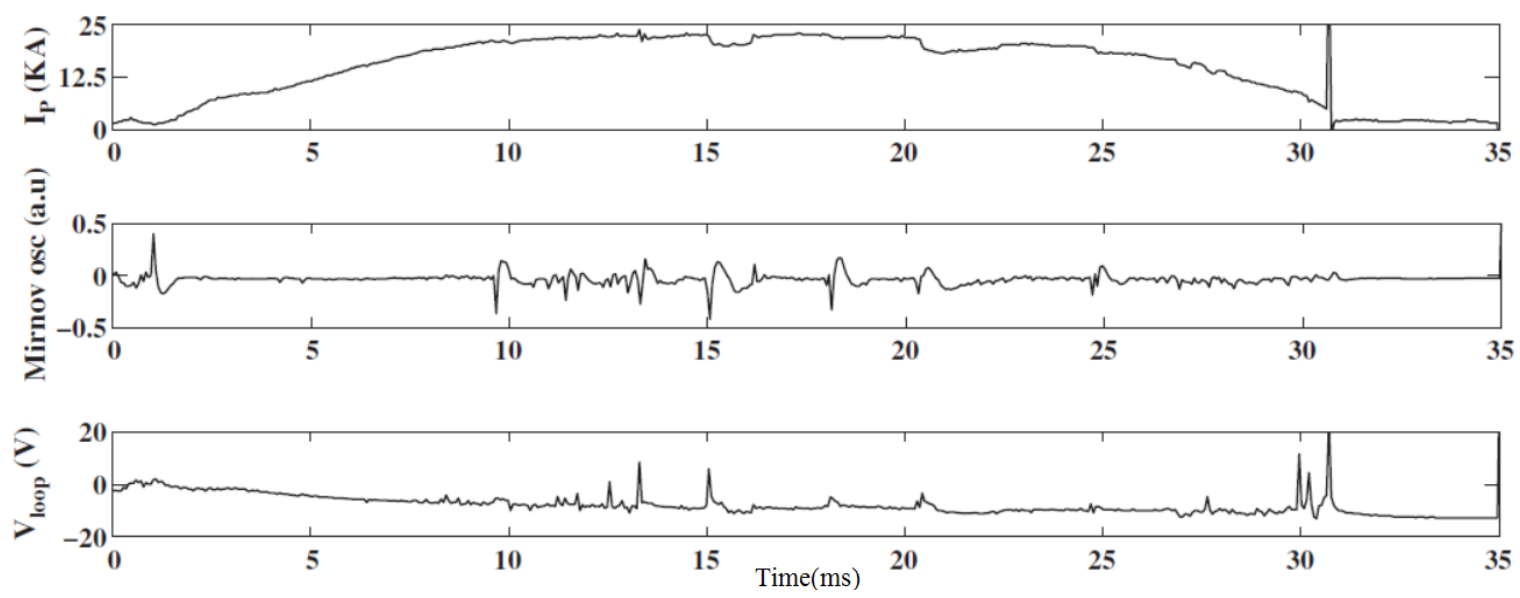

Fig. 5. Time evolution of $I_{p}$, MO and loop voltage in the absence of HLB and RHF.
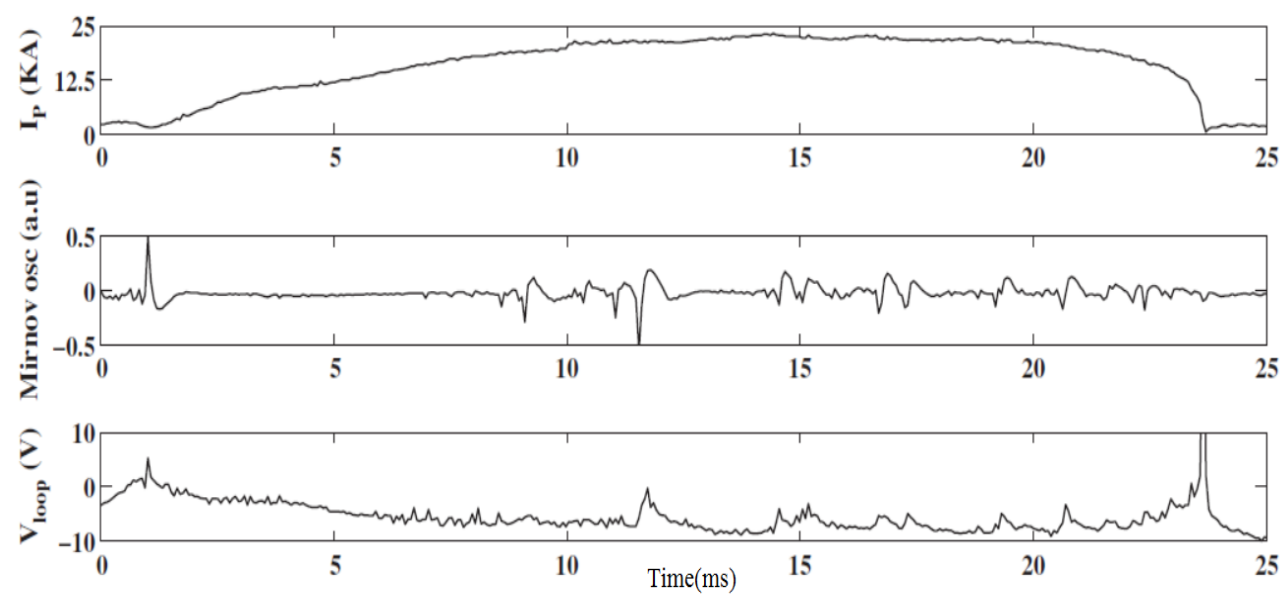

Fig. 6. Time evolution of $I_{p}$, MO, loop voltage and in the presence of the positive Emissive Biased Limiter $(E B L)\left(V_{b}=+250 V\right)$. The bias voltage $\left(\mathrm{V}_{\mathrm{b}}\right)$ is applied at $\approx 10 \mathrm{~ms}$ for $(\mathrm{r} / \mathrm{a}) \& \mathrm{LB}=0.92$.

of the $M O$ in emissive positive polarity is higher and more regular than the normal state.

\section{RHF setup for IR-T1 and its parameters measurement}

In this work, regarding the MIs in tokamak including the diagnostics and the external rotation control system, the main characteristics of plasma were investigated. In fact, specific perturbations were typically generated by the MIs regardless of their type $(2 / 1,3 / 2,3 / 1$, etc.). Furthermore, there is a resonant interaction between the MIs structure and the external fields having a matching structure. Therefore, the external control of the GR would be allowed by inducing a rotating Resonant Magnetic Perturbation (RMP) on the plasma. It should be noted that the MIs rotation, changes corresponding to the rotation rate of the $R M P$. The $R M P$ would be generated by the structure of external coils. These coils are positioned above the insulating quartz in the vacuum chamber. Therefore, the field's frequency would be able to penetrate into the plasma despite the fact that they utilize the coils outside the conducting vacuum chamber.

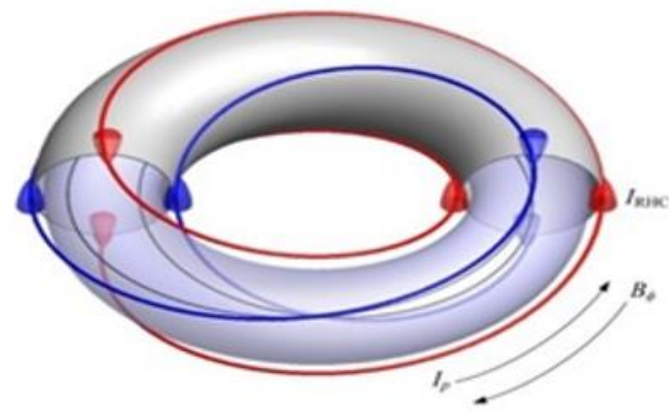

Fig. 7. $R H F$ coils ( $\mathrm{L}=2 \& 3$ modes) on the outer surface of the IR-T1 chamber.

Such a system could efficiently alter the frequency of the $M I s$ as reported by different experiments. In most of tokamaks, if the $R H F$ is applied, the $M H D$ behavior could be controlled. The RHF has been known as an external helical magnetic field being capable of enhancing the plasma confinement in tokamak. In the IR-T1, this field is generated by two coils wrapped around the tokamak chamber externally having a 
Table 2. Modes and $W$ by applying $H L B$ in 8-12.2 ms.

\begin{tabular}{|c|c|c|c|c|c|c|c|c|c|c|c|c|c|c|}
\hline Time (ms) & $\begin{array}{l}8- \\
8.3\end{array}$ & $\begin{array}{l}8.3- \\
8.6\end{array}$ & $\begin{array}{l}8.6- \\
8.9\end{array}$ & $\begin{array}{l}8.9- \\
9.2\end{array}$ & $\begin{array}{l}9.2- \\
9.5\end{array}$ & $\begin{array}{l}9.5- \\
9.8\end{array}$ & $\begin{array}{l}9.8- \\
10.1\end{array}$ & $\begin{array}{l}10.1- \\
10.4\end{array}$ & $\begin{array}{l}10.4- \\
10.7\end{array}$ & $\begin{array}{l}10.7- \\
11\end{array}$ & $\begin{array}{l}11- \\
11.3\end{array}$ & $\begin{array}{l}11.3- \\
11.6\end{array}$ & $\begin{array}{l}11.6- \\
11.9\end{array}$ & $\begin{array}{l}11.9 \\
12.2\end{array}$ \\
\hline $\mathrm{m}$ & 3 & 3 & 3 & 3 & 3 & 4 & 3 & 2 & 2 & 2 & 3 & 3 & 4 & 2 \\
\hline $\mathrm{W}(\mathrm{cm})$ & 3.19 & 3.16 & 3.14 & 3.11 & 2.08 & 3.06 & 3.03 & 3.01 & 2.28 & 2.26 & 2.23 & 2.93 & 3.09 & 2.60 \\
\hline$\frac{d w}{d t} \times 10^{4}\left(\frac{\mathrm{cm}}{\mathrm{s}}\right)$ & 1.06 & 1.05 & 1.04 & 1.03 & 1.02 & 1.01 & 1 & 0.76 & 0.75 & 0.74 & 0.97 & 1.03 & 0.91 & 0.86 \\
\hline
\end{tabular}

Table 3. Modes and $W$ by applying the $R H F(\mathrm{~L}=2)$ in $8-12.2 \mathrm{~ms}$.

\begin{tabular}{|c|c|c|c|c|c|c|c|c|c|c|c|c|c|c|}
\hline Time (ms) & $\begin{array}{l}8- \\
8.3\end{array}$ & $\begin{array}{l}8.3- \\
8.6\end{array}$ & $\begin{array}{l}8.6- \\
8.9\end{array}$ & $\begin{array}{l}8.9- \\
9.2\end{array}$ & $\begin{array}{l}9.2- \\
9.5\end{array}$ & $\begin{array}{l}9.5- \\
9.8\end{array}$ & $\begin{array}{l}9.8- \\
10.1\end{array}$ & $\begin{array}{l}10.1- \\
10.4\end{array}$ & $\begin{array}{l}10.4- \\
10.7\end{array}$ & $\begin{array}{l}10.7- \\
11\end{array}$ & $\begin{array}{l}11- \\
11.3\end{array}$ & $\begin{array}{l}11.3- \\
11.6\end{array}$ & $\begin{array}{l}11.6- \\
11.9\end{array}$ & $\begin{array}{l}11.9 \\
12.2\end{array}$ \\
\hline $\mathrm{m}$ & 4 & 2 & 2 & 3 & 2 & 3 & 3 & 2 & 2 & 2 & 2 & 4 & 2 & 3 \\
\hline $\mathrm{W}(\mathrm{cm})$ & 4.39 & 2.61 & 2.59 & 3.36 & 2.55 & 3.30 & 3.26 & 2.49 & 2.47 & 3.18 & 2.41 & 3.98 & 2.36 & 3.04 \\
\hline$\frac{d w}{d t} \times 10^{4}\left(\frac{\mathrm{cm}}{\mathrm{s}}\right)$ & 1.46 & 0.87 & 0.86 & 1.12 & 0.85 & 1.1 & 1.8 & 0.83 & 0.82 & 1.06 & 0.80 & 1.32 & 0.78 & 1.01 \\
\hline
\end{tabular}

specified rotation direction. The minor radius (a) of these $H C s$ are $21 \mathrm{~cm}(\mathrm{~L}=2, \mathrm{n}=1)$ and $22 \mathrm{~cm}(\mathrm{~L}=3, \mathrm{n}=1)$ whereas the major radius (R) is $50 \mathrm{~cm}$ (Fig. 7). In this experiment, the current through the $H C s$ was in the range 200 - 300 A much lower than $I_{p}$ (32 kA). Fig. 3 shows

the RHF coils' positions. It should be noted that $M H D$ oscillations could be due to the $R H F$ effect on the plasma discharge.

The effects of the $R H F$ on the IR-T1 plasma discharge in presence of $\mathrm{L}=2 \& 3$ modes have been investigated [12]. It was found that in the presence of the $R H F$, particularly for $\mathrm{L}=2 \& 3$, plasma parameters could enhance the confinement time by flatting $I_{p}$.

Meanwhile, the poloidal components of the $M O$ in the absence and presence of the $R H F$ were compared [12]. To avoid multiplicity of images, only some oscillations of the coils $\left(p_{1}=0^{\circ}, p_{3}=90^{\circ}, p_{5}=180^{\circ}\right.$ and $\left.p_{7}=270^{\circ}\right)$ were investigated. As can be seen, the best way to reduce the magnetic oscillations is using the $R H F$ with $\mathrm{L}=2$.

\section{Results and discussion}

\subsection{Magnetic islands width and their growth rate}

Based on the definition of safety factor $(q=m / n$ and considering that in IR-T1, the toroidal mode number $n=1$, then by finding $m, q$ can be obtained at the rational surfaces. The explosive $G R$ leads to their overlapping and the $M F$ lines become stochastic. To determine $W$, the following approximations can be used [13]:

$$
\frac{w}{r_{s}}=2 \sqrt{\frac{2}{m}\left(\frac{r_{c}}{r_{s}}\right)^{m} \frac{\partial B_{\theta}}{B_{\theta}}},
$$

In which $r_{s}$ refers to the radius of the rational surface due to a given mode and can be evaluated using $q=m / n=5 \times 10^{6} \times B_{T} r_{s}^{2} / I_{p} R$. Also, $r_{c}=18 \mathrm{~cm}$ is the radius of the $M C s, m$ refers to the poloidal mode number whose value can be obtained through the polar diagrams.
The quantity $\partial B_{\theta} / B_{\theta}$ can be obtained for different time intervals. Assuming that the plasma is axial, the unperturbed Poloidal Field $(P F), B_{\theta}$ can be derived using the Ampere's law. Since, $I_{p}$ is time dependent, then $B_{\theta}$ is also time dependent and in turn, the $G R$ of the $W$ can be derived by $d W / d t=W / t_{g}$, where, $t_{g}$ refers to the growth time. In tables (2-5), regard to the limitation of equation 4 [13], the modes and $W$ were compared in time interval $8-12.2 \mathrm{~ms}$ which is divided to 14 lower intervals, using the $H L B \mathrm{~s}$ and the $R H F(\mathrm{~L}=2$, $\mathrm{L}=3, \mathrm{~L}=2,3$ ).

The time interval of $8-12.2 \mathrm{~ms}$ was divided into 14 smaller equal steps of $300 \mu \mathrm{s}$. The results show that the highest repetition of modes $\mathrm{m}=4, \mathrm{~m}=3$ and $\mathrm{m}=2$ has occurred for $R H F(\mathrm{~L}=2,3), R H F(\mathrm{~L}=3)$ and $H L B$, respectively. Comparing $W$, it is observed that the $H L B$ and the RHF ( $\mathrm{L}=2)$ have lower values. On the other hand, in the most cases, the resonant modes occur in lower modes such as $2 \& 3$. Thus, the RHF $(\mathrm{L}=2)$ can be improved to maintain the confinement process. As shown in fig. 8, the Max and Min recorded Ws for $H L B$ and RHF (L=2) are $4.39 \mathrm{~cm}$ and $2.23 \mathrm{~cm}$ respectively. The most overlapping lines were at the intervals of 3-4 and 5-6, which are about 8.6-8.9 and 9.2-9.5 ms. It can be seen from fig. 9 that the highest acceleration is related to the $H L B$ while the lowest acceleration is related to the $R H F(\mathrm{~L}=3)$. Comparison of lines shows that the $R H F$ $(\mathrm{L}=2)$ has more stable variations. This issue suggests that the modes are more stable in this case.

\section{Conclusion}

One of the main reasons for developing tokamaks is to generate energy for the mankind through the nuclear fusion process. In this regard, the more time the plasma is confined, the more energy will be obtained. Two ways 
Table 4. Modes and $W$ by applying the $R H F(\mathrm{~L}=3)$ in $8-12.2 \mathrm{~ms}$.

\begin{tabular}{|c|c|c|c|c|c|c|c|c|c|c|c|c|c|c|}
\hline Time (ms) & $\begin{array}{l}8- \\
8.3\end{array}$ & $\begin{array}{l}8.3- \\
8.6\end{array}$ & $\begin{array}{l}8.6- \\
8.9\end{array}$ & $\begin{array}{l}8.9- \\
9.2\end{array}$ & $\begin{array}{l}9.2- \\
9.5\end{array}$ & $\begin{array}{l}9.5- \\
9.8\end{array}$ & $\begin{array}{l}9.8- \\
10.1\end{array}$ & $\begin{array}{l}10.1- \\
10.4\end{array}$ & $\begin{array}{l}10.4- \\
10.7\end{array}$ & $\begin{array}{l}10.7- \\
11\end{array}$ & $\begin{array}{l}11- \\
11.3\end{array}$ & $\begin{array}{l}11.3- \\
11.6\end{array}$ & $\begin{array}{l}11.6- \\
11.9\end{array}$ & $\begin{array}{l}11.9 \\
12.2\end{array}$ \\
\hline $\mathrm{m}$ & 4 & 3 & 3 & 4 & 3 & 3 & 3 & 3 & 3 & 3 & 2 & 4 & 2 & 3 \\
\hline $\mathrm{W}(\mathrm{cm})$ & 3.58 & 2.89 & 2.87 & 3.23 & 2.98 & 3.11 & 3.07 & 2.85 & 2.83 & 3.01 & 2.66 & 3.55 & 2.71 & 2.92 \\
\hline$\frac{d w}{d t} \times 10^{4}\left(\frac{\mathrm{cm}}{\mathrm{s}}\right)$ & 1.31 & 0.96 & 0.95 & 1.07 & 0.99 & 1.03 & 1.02 & 0.95 & 0.94 & 1 & 0.69 & 1.18 & 0.90 & 0.97 \\
\hline
\end{tabular}

Table 5. Modes and $W$ by applying the $R H F(\mathrm{~L}=2,3)$ in $8-12.2 \mathrm{~ms}$.

\begin{tabular}{|c|c|c|c|c|c|c|c|c|c|c|c|c|c|c|}
\hline Time (ms) & $\begin{array}{l}8- \\
8.3 \\
\end{array}$ & $\begin{array}{l}8.3- \\
8.6\end{array}$ & $\begin{array}{l}8.6- \\
8.9 \\
\end{array}$ & $\begin{array}{l}8.9- \\
9.2\end{array}$ & $\begin{array}{l}9.2- \\
9.5 \\
\end{array}$ & $\begin{array}{l}9.5- \\
9.8\end{array}$ & $\begin{array}{l}9.8- \\
10.1\end{array}$ & $\begin{array}{l}10.1- \\
10.4\end{array}$ & $\begin{array}{l}10.4- \\
10.7 \\
\end{array}$ & $\begin{array}{l}10.7- \\
11\end{array}$ & $\begin{array}{l}11- \\
11.3 \\
\end{array}$ & $\begin{array}{l}11.3- \\
11.6\end{array}$ & $\begin{array}{l}11.6- \\
11.9\end{array}$ & $\begin{array}{l}11.9 \\
12.2 \\
\end{array}$ \\
\hline $\mathrm{m}$ & 4 & 3 & 3 & 4 & 3 & 4 & 4 & 3 & 3 & 3 & 2 & 4 & 2 & 3 \\
\hline $\mathrm{W}(\mathrm{cm})$ & 3.88 & 2.99 & 2.83 & 3.14 & 2.88 & 3.13 & 3.19 & 2.91 & 2.79 & 3.04 & 2.59 & 3.61 & 2.75 & 2.94 \\
\hline$\frac{d w}{d t} \times 10^{4}\left(\frac{\mathrm{cm}}{\mathrm{s}}\right)$ & 1.32 & 0.99 & 0.94 & 1.04 & 0.96 & 1.04 & 1.06 & 0.97 & 0.93 & 1.01 & 0.86 & 1.20 & 0.91 & 0.98 \\
\hline
\end{tabular}

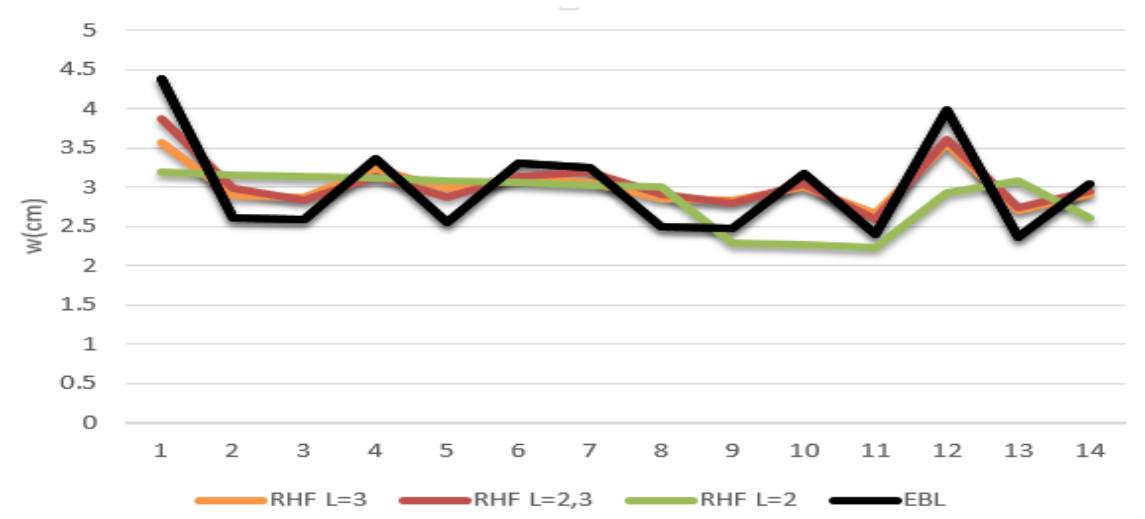

Fig. 8. Comparison of $W$ in the time interval $8-12.2$ ms divided into 14 smaller equal steps of $300 \mu$ s.

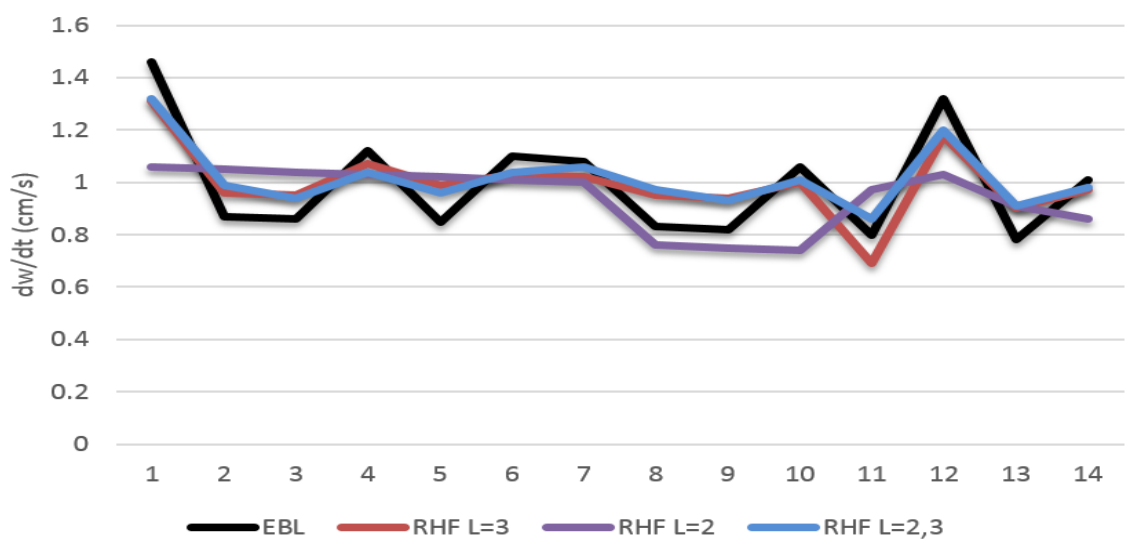

Fig. 9. Comparison of $G R$ of the $W$ in the time interval of $8-12.2 \mathrm{~ms}$ divided into 14 smaller equal steps of $300 \mu \mathrm{s}$.

of increasing confinement time are using $H L B$ and the $R H F$. One of them creates $E_{r}$ which produces a shear velocity. Another is using the $R H F$ which can be generated using two coils wrapped around the tokamak chamber externally. As was shown, by applying the $R H F, m$ was increased which resulted to the increase of safety factor. This issue in turn makes the tokamak more stable. By using different diagnostic tools installed in the IR-T1 including the $R C, M C s$ and $W$, various parameters 
of plasma were measured and compared in different conditions. Finally, $W$ and their $G R$ were obtained and compared in various situations. The results showed that

\section{References}

1. A Sykes and J A Wesson, Phys. Rev. Lett. 44 (1980) 1215.

2. S V Mirnov and I B Semenov, Sovier Atomic Energy 30 (1971) 22.

3. S Von Goeler, W Stodiek, and N Sauthoff, Phys. Rev. Lett. 33 (1974) 1201.

4. F Karger, H Wobig, S Corti, J Gernhardt, O Kluber, G Lisitano, K Mccormick, D Meisel, and S Sesnic, $5^{\text {th }}$ Conference Proceedings Tokyo, 11 (1974).

5. B V Waddell, B Carreras, H R Hicks, J A Holmes, and D K Lee, Phys. Rev. Lett. 41 (1978) 1386.

6. J J Ellis, A A Howling, A W Morris, and D C Robinson, $10^{\text {th }}$ Int. Conf. on Plasma Physics and Controlled Nuclear Fusion, London, UK, 1 (1984) 363.

7. $\mathrm{T}$ C Hender, $\mathrm{R}$ Fitzpatrick, A W Morris, P G Carolan, R D Durst, T Edlington, J Ferreira, S J the $R H F(\mathrm{~L}=2)$ has more stable variations, indicating that the modes is more stable in this case.

Fielding, P S Haynes, J Hugill, I J Jenkins, R J La Haye, B J Parham, D C Robinson, T N Todd, M Valovic, and G Vayakis, Nucl. Fusion 32 (1992) 2091.

8. M S T Araujo, A Vannucci, I L Caldas, and I L Nuovo Cimento D 18 (1996) 807.

9. E J Strait, E D Fredrickson, J M Moret, and M Takechi, Fusion Sci. Technol. 53 (2006) 304.

10. V S Mukhovatov and V D Shafranov, Nucl. Fusion 11 (1971) 605.

11. S H Seo, Phys. Plasmas 16 (2009) 032501.

12. H Noor Mohamadigh, A Salar Elahi, and M Ghoranneviss, Journal of Nuclear and Particle physics 4, 5 (2014) 142.

13. M Ghoranneviss, A Hogabri, and St Kuhn, Nucl. Fusion 43 (2003) 210. 